\title{
Engineering Mannitol Biosynthesis in Escherichia coli and Synechococcus sp. PCC 7002 Using a Green Algal Fusion Protein
}

\author{
Mary Ann Madsen, ${ }^{\dagger}$ Stefan Semerdzhiev, ${ }^{\dagger}$ Anna Amtmann, ${ }^{\dagger}$ and Thierry Tonon* ${ }^{*} \neq \odot$ \\ ${ }^{\dagger}$ Institute of Molecular, Cell, and Systems Biology, College of Medical, Veterinary, and Life Sciences, University of Glasgow, \\ Glasgow G12 8QQ United Kingdom \\ ${ }^{\ddagger}$ Centre for Novel Agricultural Products, Department of Biology, University of York, Heslington, York YO10 5DD, United Kingdom
}

Supporting Information

ABSTRACT: The genetic engineering of microbial cell factories is a sustainable alternative to the chemical synthesis of organic compounds. Successful metabolic engineering often depends on manipulating several enzymes, requiring multiple transformation steps and selection markers, as well as protein assembly and efficient substrate channeling. Naturally occurring fusion genes encoding two or more enzymatic functions may offer an opportunity to simplify the engineering process and to generate ready-made protein modules, but their functionality in heterologous systems remains to be tested. Here we show that heterologous expression of a fusion enzyme from the marine alga Micromonas pusilla, comprising a mannitol-1-phosphate dehydrogenase and a mannitol-1-phosphatase, leads to synthesis of mannitol by Escherichia coli and by the cyanobacterium Synechococcus sp. PCC 7002. Neither of the heterologous systems naturally produce this sugar alcohol, which is widely used in food, pharmaceutical, medical, and chemical industries. While the mannitol production rates obtained by single-gene manipulation were lower than those previously achieved after pathway optimization with multiple genes, our findings show that naturally occurring fusion proteins can offer simple building blocks for the assembly and optimization of recombinant metabolic pathways.

KEYWORDS: synthetic biology, fusion protein, mannitol, cyanobacteria, Micromonas
$\mathrm{M}$ icrobial cell factories, particularly photosynthetic chassis that consume carbon dioxide as their sole carbon source, are an attractive alternative to chemical synthesis. ${ }^{1}$ They present a sustainable approach to producing an array of substances with usages in food, pharmaceutical, nutraceutical, and cosmetic industries.

Biological production platforms struggle to compete economically, particularly for the production of low value commodity chemicals and materials. ${ }^{2}$ A recurring problem is performance, which is measured as titer, yield, and productivity. Artificial metabolic pathways inherently generate imbalances in pathway flux resulting in (toxic) intermediate and side product accumulation, growth inhibition, and ultimately low product yield. ${ }^{3}$ Therefore, individual modifications (e.g., the introduction of a single enzyme or pathway) are not sufficient to achieve industrially relevant titers. As a result, cell factory development requires the introduction of several genes to synthesize the desired product, the overproduction of precursors, and the deletion of competing pathways. ${ }^{4,5}$

The use of fusion genes, generated from previously separate genes, can help to simplify the biological engineering process. ${ }^{6}$ First, reducing the number of genes required for a synthetic pathway simplifies the design and assembly of synthetic expression constructs as well as the transformation and selection of producing strains. Second, reducing the number of enzymes simplifies assembly of protein complexes encoded by separate genes and stoichiometric optimization to balance pathway flux. Furthermore, catalytic sites are brought in closer proximity, thus enhancing substrate channeling and reducing intermediate accumulation and/or loss to competing pathways. ${ }^{7}$ Additionally, transit times for intermediate products between catalytic sites are significantly reduced, thus improving biosynthetic efficiency.

D-Mannitol is a sugar alcohol, or polyol, ubiquitous in prokaryotes and eukaryotes, except for Archaea and animals. It has desirable properties for food, pharmaceutical, medical, and chemical industries. ${ }^{8}$ On the basis of a recent analysis of mannitol market (http://www.grandviewresearch.com/ industry-analysis/mannitol-market), demand for this polyol, driven by an increasing use in the food and pharmaceutical sectors, is expected to reach 35.9 million $\mathrm{kg}$ representing a market of USD 418.3 million by 2024 .

Mannitol can be produced by extraction, chemical synthesis, or biosynthesis. Traditionally, mannitol was harvested from plant material that was seasonal and had highly variable yields. ${ }^{9}$ To stabilize mannitol supplies, commercial production now mostly occurs through chemical hydrogenation of fructoseglucose syrups. However, poor selectivity of the nickel catalyst results in a mixture of mannitol and sorbitol that is relatively difficult to separate and thus costly. ${ }^{10}$ Chemical synthesis can be

Received: June 7, 2018

Published: November 8, 2018 
improved by altering the substrate, e.g., isomerizing glucose to fructose by enzymatic conversion; however, enzyme availability and added costs of additional steps prevent this from being economical.

The chemical industry is now looking to biobased production methods to reduce costs and environmental impact. The most successful approach to mannitol biosynthesis currently uses heterofermentative lactic acid bacteria. Under anaerobic conditions, these organisms reduce fructose using the native enzyme mannitol dehydrogenase. This approach requires an external sugar supply that is (a) predominantly obtained from traditional crops, e.g., corn, and (b) a major cost for biosynthesis of commodities such as mannitol. External carbon sources and associated costs can be eliminated from the bioproduction pipeline by using photosynthetic organisms, which assimilate atmospheric carbon dioxide into sugars via the Calvin cycle. ${ }^{11}$

Cyanobacteria represent an incredibly diverse phylum of phototrophic prokaryotes that are being developed for photosynthetic bioproduction. ${ }^{12-14}$ One particularly attractive chassis is the unicellular euryhaline cyanobacterium Synechococcus sp. PCC 7002 due to its reported fast growth and tolerance of high salt, light, and temperature. ${ }^{15-17}$ Importantly, Synechococcus is amenable to natural transformation, ${ }^{18,19}$ and its genome is fully sequenced (http://genome.microbedb.jp/cyanobase/ SYNPCC7002). Some molecular tools to engineer Synechococcus have been developed including transformation vectors, promoters to control transcription, and ribosome binding sites to control translation. ${ }^{20-22}$

Synechococcus has previously been engineered to produce mannitol from fructose-6-phosphate, an intermediate of the Calvin cycle. ${ }^{23}$ This recombinant mannitol biosynthetic pathway involved heterologous expression of two enzymes encoded by separate genes: (1) an Escherichia coli gene encoding mannitol-1-phosphate dehydrogenase (M1PDH), which catalyzes the reduction of fructose-6-phosphate (F6P) to mannitol1-phosphate (M1P), and (2) an Eimeria tenella gene encoding phosphohistidine mannitol-1-phosphatase (M1Pase), which hydrolyses M1P to mannitol.

In recent years, new insights have been gained on the mannitol biosynthetic pathway, notably in algae. The first algal genes involved in mannitol production were identified in the model brown alga Ectocarpus, ${ }^{24}$ and biochemical characterization of the recombinant enzymes confirmed M1PDH and M1Pase function. ${ }^{25-27}$ Analysis of the distribution and evolution of these mannitol biosynthetic genes across algal lineages showed that mannitol synthesis is more widely spread and diverse that initially thought. ${ }^{28}$ Interestingly, several fusion genes combining modules for M1PDH and M1Pase activities were identified, notably in marine green algae. For instance, Mipuc10g00620 (http://bioinformatics.psb.ugent.be/orcae/ annotation/Mipuc/current/Mipuc10g00620) of Micromonas pusilla strain CCMP1545 is predicted to encode an enzyme expected to transform F6P directly into mannitol.

In this study, we explored whether the M1PDH/M1Pase fusion gene of M. pusilla strain CCMP1545 is functional when heterologously expressed in heterotrophic (E. coli) or photoautotrophic (Synechococcus) bacteria. To facilitate the engineering of Synechococcus we generated BioBrick-compatible molecular tools (vectors and ribosome binding sites). We found that both E. coli and Synechococcus produced mannitol when transformed with the fusion gene. The one-step mannitol production pathway provides an excellent starting point for further optimization of sustainable mannitol production in cyanobacteria.

\section{RESULTS}

Mpusfus Is Functional in E. coli. The M1PDH/M1Pase fusion gene of Micromonas pusilla strain CCMP1545 was codon optimized for expression in Synechococcus (see Methods), and we called the resulting sequence mpusfus. To test expression and function in heterologous systems, mpusfus was first introduced into E. coli using the pFO4 vector that carries an IPTG-inducible expression system. A six-histidine tag was added to the $5^{\prime}$ end of the gene. Western blot of protein extracts from IPTG-induced cells revealed a band of the expected size, while no band was detected in the controls without IPTG (Figure 1). The size of

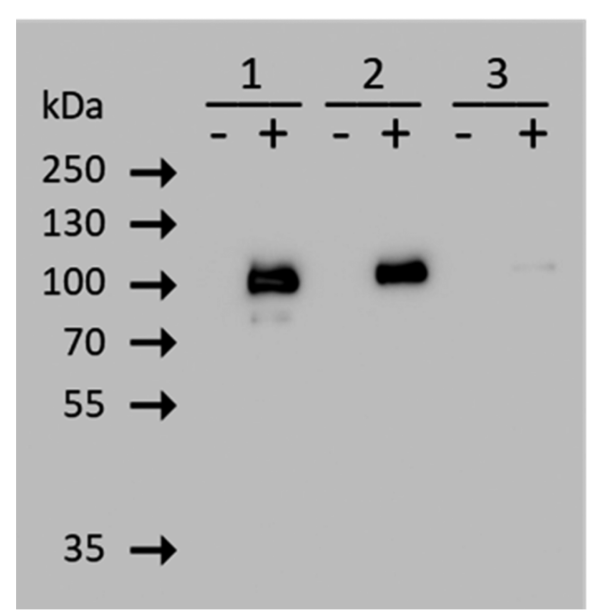

Figure 1. Expression of the M1PDH/M1Pase fusion protein in E. coli. Western blot analysis of His-tagged M1PDH/M1Pase in soluble fractions of protein extracts from E. coli transformed with mpusfus. Lanes marked 1, 2, and 3 contain protein extracts from three independent clones after culture in absence $(-)$ and in presence $(+)$ of IPTG. Numbers at the left of the panel indicate size $(\mathrm{kDa})$ of proteins in the ladder.

the primary band was close to the value of $94.8 \mathrm{kDa}$ calculated for the full-length predicted amino acid sequence. This showed that a single fusion protein was produced from the fusion gene.

Mannitol was measured both in the culture media and in the cells after $20 \mathrm{~h}$ of incubation in absence and in the presence of IPTG. In $5 \mathrm{~mL}$ cultures with a final $\mathrm{OD}_{600}$ of 2.5-3.0 we found 1 $\pm 0.05 \mathrm{mg}$ of mannitol in the media and $0.08 \pm 0.008 \mathrm{mg}$ of mannitol inside the cells ( $n=3$ clones) after incubation with IPTG. Extrapolated to a $1 \mathrm{~L}$ culture, total mannitol production was $218 \pm 11.9 \mathrm{mg} / \mathrm{L}$, of which $202 \pm 10.7 \mathrm{mg}$ (93\%) were exported into the medium and $16 \pm 1.6 \mathrm{mg}(7 \%)$ were retained inside the cells. No mannitol was detected in cultures grown without IPTG. The results showed that the algal fusion gene produces a single protein in E. coli that catalyzes the biosynthesis of mannitol.

New Molecular Tools and Protocols To Engineer Synechococcus sp. PCC 7002. BioBricks represent the largest collection of standardized parts for genetic engineering. ${ }^{29}$ While a number of genetic tools have been developed for Synechococcus, ${ }^{20-22}$ none of them are compatible with BioBrick parts. In order to develop a BioBrick-compatible integration vector for Synechococcus, we reengineered an existing expression vector, $\mathrm{pAQ} 1 \mathrm{EX} .^{30}$ This vector targets the transgene to a neutral site in the native, high copy pAQ1 plasmid of Synechococcus. 
BioBrick prefix and suffix sequences containing the restriction enzyme recognition sites required for gene assembly were synthesized and inserted into the integrative region of the pAQ1EX vector. For ease of cloning, domestication of DNA parts is important, whereby BioBrick restriction sites do not occur outside of the prefix and suffix sequences. An XbaI site occurring directly upstream of the selective aadA gene was therefore replaced with an XhoI site. Finally, the phycocyanin promoter of Synechocystis sp. PCC 6803, $\mathrm{P}_{\mathrm{cpcB} 594}$, which has been reported to be a strong promoter in cyanobacteria, was inserted into the BioBrick cloning site. ${ }^{31}$ The resulting vector called pAQ1BB provides a convenient tool for transformation of Synechococcus sp. PCC 7002 (Figure 2). The target integration

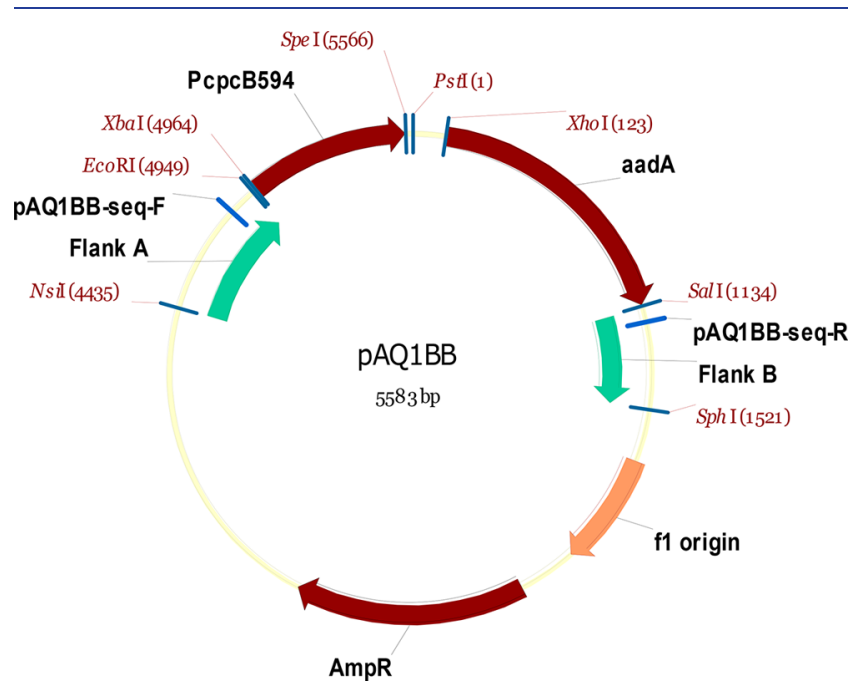

Figure 2. New BioBrick vector for transformation of Synechococcus sp. PCC 7002. Region between Flanks A and B stably integrates into the native pAQ1 plasmid of Synechococcus by homologous recombination. This region includes a spectinomycin resistance gene $(\operatorname{aad} A)$ and the BioBrick cloning site containing the phycocyanin promoter of Synechocystis sp. PCC $6803\left(\mathrm{P}_{\mathrm{cpcB} 594}\right)$.

site ("landing pad") can be modified by replacing homologous sequences Flank A and Flank B using NsiI/EcoRI and SalI/SphI restriction sites, respectively. The marker gene for selection can be modified using XhoI and SalI restriction sites.

Using established protocols for natural transformation, ${ }^{30}$ transformation efficiency of Synechococcus with pAQ1BB was initially poor. Increasing both the amount of DNA and incubation time prior to plating onto selective media significantly enhanced transformation efficiency (Figure S1). The optimized transformation protocol can be found in the Methods section.

Ribosome binding sites (RBSs) play an essential role in translation initiation during protein synthesis. Software have been developed to design synthetic RBSs considering factors such as secondary mRNA structure influenced by flanking nucleotide sequences, the Shine-Dalgarno (SD) sequence complementary to the $16 \mathrm{~S}$ rRNA, spacing between the SD and start codon, and the sequence of the start codon itself. ${ }^{32}$ We used a web-based RBS calculator ${ }^{33}$ to design RBS for Synechococcus (sequences in Table S2) and assessed the suitability of three potential RBS for producing green fluorescent protein (GFP). RBS-GFP were cloned into $\mathrm{pAQ} 1 \mathrm{BB}$ and used to transform Synechococcus. Similar to a previous report, ${ }^{21}$ we observed a moderate correlation between predicted and actual translation rates (Figure 3). Both the RBS calculator and the GFP-assay identified RBS3 as the strongest RBS, and we therefore used RBS3 to express mpusfus.

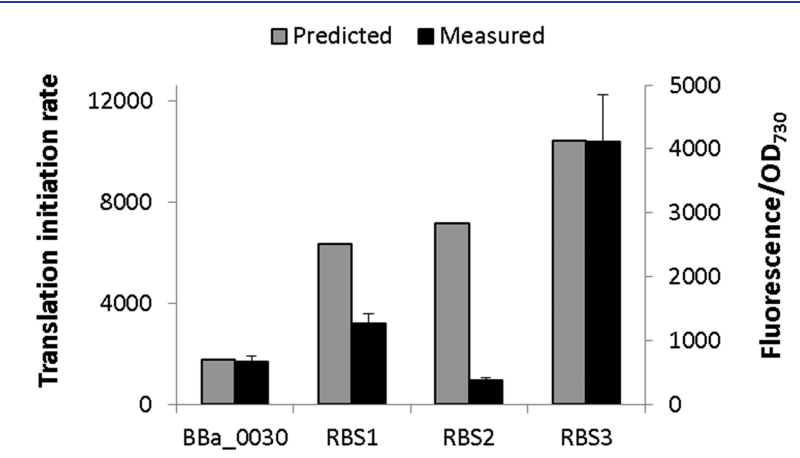

Figure 3. Predicted and measured strength of synthetic ribosome binding sites for Synechococcus sp. PCC 7002. Gray bars represent translation initiation rates predicted in silico using RBS calculator. Black bars represent GFP fluorescence normalized to cell density (optical density at $730 \mathrm{~nm}, \mathrm{OD}_{730}$ ). Each RBS-GFP construct was expressed in Synechococcus under the control of the $\mathrm{P}_{\mathrm{cpcB5} 54}$ promoter and GFP fluorescence was measured when culture density reached an $\mathrm{OD}_{730}$ of 1 . Data are presented as means \pm SEM of three independent cultures. BBa_0030 is a standard RBS from the Registry of Standardised Parts. $\mathrm{RBS1}, \mathrm{RBS} 2$, and RBS3 were forward engineered using RBS calculator.

To assess promoter activity in combination with RBS3, we monitored GFP fluorescence in the $\mathrm{P}_{\mathrm{cpcB} 594}$ :RBS3-GFP expressing cells over the course of culture growth. The experiment showed that the promoter is most active in the early exponential phase (Figure 4 ) as previously suggested. ${ }^{30}$

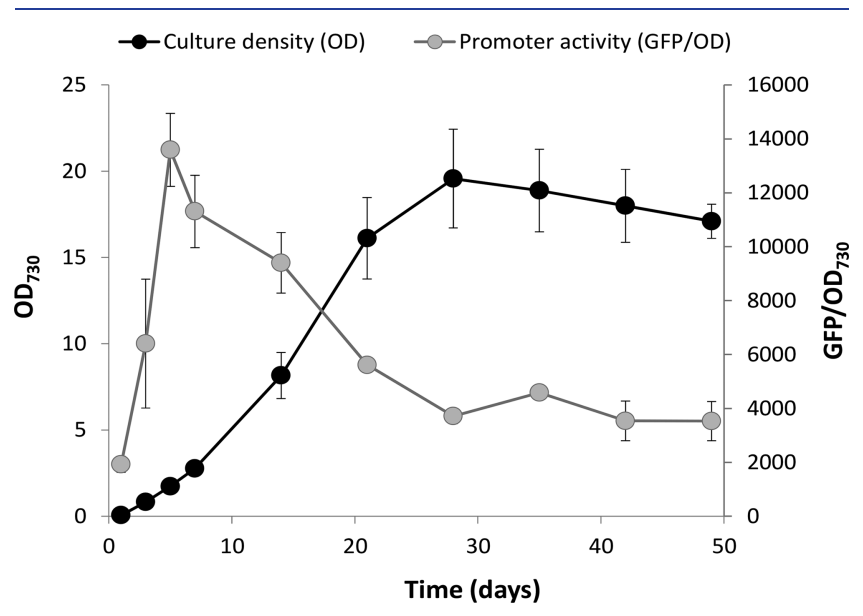

Figure 4. Promoter activity over the course of culture growth. Black points represent culture density $\left(\mathrm{OD}_{730}\right)$. Gray points represent GFP fluorescence normalized to cell density $\left(\mathrm{GFP} / \mathrm{OD}_{730}\right)$. RBS3-GFP was expressed in Synechococcus under the control of the $\mathrm{P}_{\mathrm{cpcB} 594}$ promoter and GFP fluorescence was measured over the course of culture growth. Data are presented as means \pm SEM of three independent cultures.

Mpusfus Is Functional in Synechococcus sp. PCC 7002. Synechococcus was transformed with the plasmid containing $\mathrm{P}_{\mathrm{cpcB594}}$ :RBS3-mpusfus. Initial growth rates were slightly impaired in the engineered Synechococcus and the densities of stationary phase cultures were slightly lower than in wild type cells (Figure 5A). Mannitol was measured in the media and in the cell pellet of wild type- and mpusfus-expressing cultures. No mannitol was detected in wild type cultures, confirming a 
A

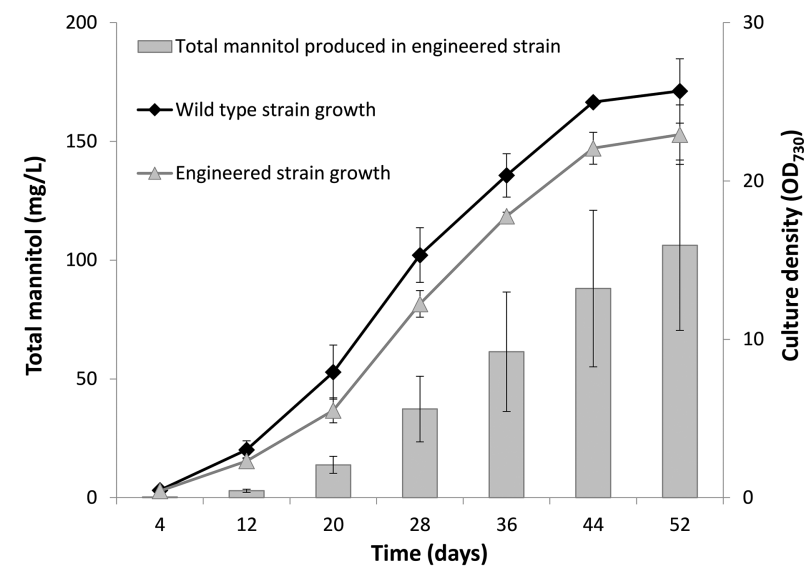

B

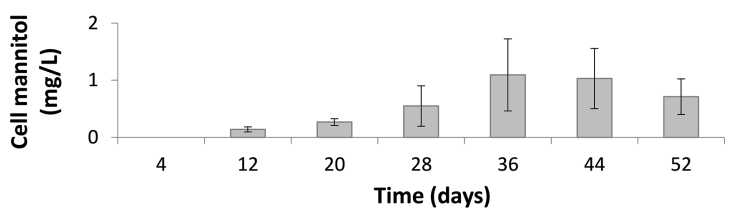

$\mathrm{C}$

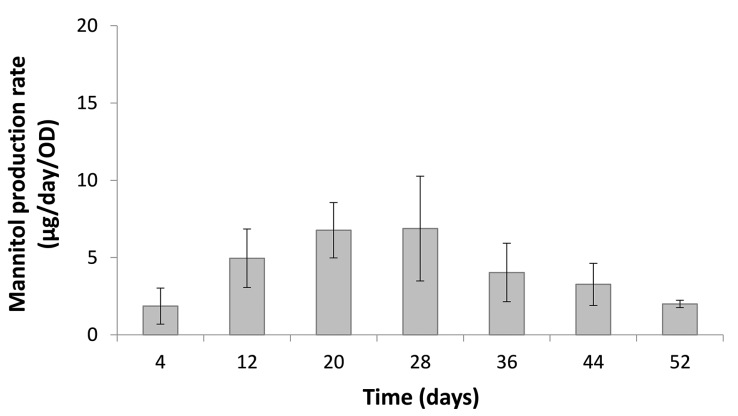

Figure 5. Mannitol production in mpusfus-expressing Synechococcus cultures. (A) Line graphs represent culture growth as optical density at $730 \mathrm{~nm}\left(\mathrm{OD}_{730}\right)$ measured over time in wild type (black symbols) and engineered Synechococcus (gray triangles) expressing mpusfus under the control of $\mathrm{P}_{\text {cpcB594 }}$ and RBS3. Gray bars represent total mannitol (sum of mannitol measured in the cellular fraction and in the medium). (B) Amount of mannitol (per $1 \mathrm{~L}$ culture) present in the cellular fraction of the engineered Synechococcus culture. (C) Mannitol production rate in the engineered Synechococcus culture normalized to $\mathrm{OD}_{730}$. All data are means \pm SEM of three independent cultures.

previous report that Synechococcus does not naturally produce mannitol. ${ }^{23}$ By contrast, mannitol was detected both in the cellular fraction and in the media of mpusfus-expressing cultures, indicating that the fusion protein is functional in Synechococcus. Total mannitol steadily increased over the course of culture growth to around $100 \mathrm{mg} / \mathrm{L}$ in early stationary phase (Figure 5A). Mannitol in the cellular fraction of the culture increased with culture growth (Figure 5B), but the majority of mannitol produced was exported into the medium. At the end of the experiment over $99 \%$ of the total mannitol was found in the culture medium.

Mannitol production rates were calculated by dividing the amount of mannitol produced between two time points by time and by mean cell number $\left(\mathrm{OD}_{730}\right)$ in this time period. As shown in Figure $5 \mathrm{C}$ the production rate increased to $7 \mu \mathrm{g} /$ day/OD on day 28 (mid-exponential phase) and then decreased.

In summary, the results show that expression of a single fusion gene encoding two enzyme functions results in the production of mannitol in heterologous systems that do not naturally synthesize mannitol.

\section{DISCUSSION}

Use of Fusion Genes for Biotechnology. Fusion genes encode more than one enzymatic function in one gene. They have potential benefits for biotechnology because they would reduce the number of constructs and transformations required for engineering metabolic pathways. Furthermore, it is hoped that, in a naturally evolved fusion protein, occurrence of adjacent modules catalyzing consecutive steps in a metabolic process will prevent loss of intermediate through efficient substrate channeling. However, functionality of fusion genes/proteins in heterologous systems remained to be proven. In this study, we show that a fusion gene from the green alga Micromonas pusilla is functional and sufficient to achieve production of mannitol in prokaryotic systems, namely E. coli and Synechococcus sp. PCC 7002.

M. pusilla strain CCMP1545 is a unicellular marine green alga that is a potentially valuable source of fusion genes for biotechnology. A high quality draft of its $22 \mathrm{Mb}$ genome is available, and resolution continues to improve around the telomeric regions of its 19 chromosomes. $^{34,35}$ Bioinformatic analyses have identified a number of fusion genes in the CCMP1545 genome encoding enzymes involved in a variety of cellular processes including pigment production, ${ }^{36}$ polyamine biosynthesis, ${ }^{37}$ DNA double-strand break repair ${ }^{38}$ and carbon fixation. ${ }^{35}$ Some Micromonas genes have been characterized by heterologous expression to date, ${ }^{39-42}$ but none of them encode fusion proteins. Therefore, the potential of the Micromonas fusion genes for metabolic engineering remained to be explored. The gene employed in this study combined a M1PDH and an M1Pase module and was tested for producing mannitol in prokaryotic model systems.

We first expressed the mpusfus fusion gene in E. coli. This organism has been previously engineered to develop whole-cell biotransformation systems for mannitol synthesis from glucose, ${ }^{43,44}$ resulting in mannitol molar yield of $80 \%{ }^{43}$ and $87 \%$. ${ }^{44}$ We found that E. coli expressing the single mpusfus fusion gene produced a single recombinant protein of approximately $100 \mathrm{kDa}$ and produced mannitol, most of which was exported into the medium. This shows that the fusion protein is functional in E. coli. However, the achieved mannitol concentration of 218 $\mathrm{mg} / \mathrm{L}$ under our experimental conditions (molar yield of $2 \%$ on glucose) was considerably lower than in the previously engineered strains. ${ }^{43,44}$ Additional manipulations addressing codon usage, metabolic flux toward substrate, substrate transport, and feeding could now be attempted to increase mannitol titers in E. coli.

Photosynthetic bacteria provide an opportunity to produce organic compounds from $\mathrm{CO}_{2}$ without the need of feeding sugars. We were therefore interested to test whether the mpusfus fusion gene can be used to produce mannitol in a photoautotrophic system. Synechococcus sp. PCC 7002 is one of the model systems for metabolic engineering of cyanobacteria and has previously been engineered to produce mannitol from F6P using two separate genes (M1PDH from E. coli and M1Pase from Eimeria tenella). ${ }^{23}$ As E. coli, Synechococcus does not possess an endogenous pathway for mannitol production. To facilitate the engineering of Synechococcus, we first generated a BioBrick compatible vector and a synthetic ribosomal binding site. The new tools in combination with a previously identified "superstrong" promoter were confirmed by GFP-assay and are available to the scientific community. 
Successful functional expression of mpusfus in Synechococcus was proven by the appearance of mannitol in the transgenic cultures. As before, ${ }^{23}$ the majority of mannitol produced was exported into the media where it can easily be harvested. Since Synechococcus does not naturally produce mannitol it is likely that the export occurs through nonspecific transport proteins for other compounds. Synechococcus and other cyanobacteria have been shown to release low-molecular-weight metabolites when subjected to hypo-osmotic stress, ${ }^{45,46}$ but the exact transport pathways remain to be identified.

The total amount of mannitol produced in this report is considerably lower than in the previous study, ${ }^{23}$ namely around $0.1 \mathrm{~g} / \mathrm{L}$ compared to $0.6 \mathrm{~g} / \mathrm{L}$. Usage of a glycogen-deficient strain helped to increase titers, ${ }^{23}$ but the main differences between the two studies lies in the growth rate of the cultures. The highest mannitol-producing strain reported previously ${ }^{23}$ reached a maximal $\mathrm{OD}_{730}$ of around 10 within $150 \mathrm{~h}$ and achieved the aforementioned mannitol concentration in $300 \mathrm{~h}$. By contrast, our strain grew much more slowly and required 50 days to produce $0.1 \mathrm{~g} / \mathrm{L}$ mannitol albeit reaching a higher $\mathrm{OD}_{730}$ of 25. It is likely that protein turnover over such a long period of time prevents the accumulation and maintenance of substantial amounts of recombinant protein. Protein synthesis then becomes very sensitive to promoter activity, which was maximal during early culture growth (see Figure 4). A combination of low promoter activity and protein turnover would explain why production rates were very low and decreased even before the cultures entered stationary phase (see Figure 5C). It can therefore be expected that usage of new promoter(s), which are active during the late stages of growth, and changes in growth conditions, e.g., fed-batch cultivation to keep cultures in the production stage for longer, could increase mannitol productivity by engineered Synechococcus sp. PCC 7002.

Despite the ability of cyanobacteria to use atmospheric carbon for industrial bioproduction, carbon availability is a key limiting factor in polyol production. The use of fast-growing strains and the development of efficient photobioreactors will be crucial to move toward industrial scale production systems that can compete with the traditional sugar-fed cultures. Our demonstration that an algal fusion gene is functional in cyanobacteria presents an important step toward simplifying the generation of recombinant metabolic pathways, and can now be combined with the usual metabolic engineering strategies to overcome metabolic bottlenecks. Metabolic network models have become available for Synechococcus, ${ }^{47}$ which should facilitate this task.

\section{METHODS}

Construction of pAQ1BB Vector for Transformation of Synechococcus sp. PCC 7002. The integrative vector pAQ1EX for transformation of Synechococcus sp. PCC $7002^{30}$ was modified to accommodate BioBrick assembly. Synthetic oligonucleotides were annealed to generate BioBrick prefix and suffix sequences flanked by restriction site overhangs for insertion into pAQ1EX using EcoRI/NcoI and NdeI/BamHI restriction sites respectively (Table $\mathrm{S} 1$ ). To prevent interference with the BioBrick assembly, an XbaI restriction site at the $5^{\prime}$ end of the spectinomycin resistance gene aadA was replaced with an XhoI restriction site by site-directed mutagenesis using primers listed in Table S1. Modifications were confirmed by sequencing, and the resulting vector was designated pAQ1BB. To drive transgene expression, the $594 \mathrm{bp}$ phycocyanin promoter of Synechocystis sp. PCC 6803, $\mathrm{P}_{\text {cpcB5944 }}{ }^{30}$ was amplified by PCR from genomic DNA using forward primer 5'-GAATTC-
GCGGCCGCTTCTAGAGTTCGTTATAAAATAAACTTAACAAATCTATAC-3' and reverse primer 5'-CTGCAGCGGCCGCTACTAGTAGGAATTAATCTCCTACTTGACTTTATG-3', and inserted into the pAQ1BB BioBrick cloning site.

Generation of Codon-Optimized mpusfus. The 863 aa $\mathrm{M} 1 \mathrm{PDH} / \mathrm{M} 1 \mathrm{Pase}$ fusion protein of Micromonas pusilla strain CCMP1545 contains codons that may reduce translation efficiency in cyanobacteria. Codon optimization for expression in Synechococcus sp. PCC 7002 was therefore performed (GenScript USA Inc.) using the OptimumGene algorithm, which takes into consideration a variety of parameters important for gene expression efficiency. These include, but are not limited to, codon usage bias, GC content, mRNA secondary structure, internal ribosome binding sites, and restriction sites. Codon usage bias is scored as a codon adaptation index (CAI), where CAI 1.0 is optimal in the desired expression organism, and CAI > 0.8 is regarded as good in terms of high expression. Following codon optimization, the CAI was improved from 0.66 in the native gene to 0.96 in the codon-optimized gene (Supporting Information and Figure S1). GC content and distribution was also optimized to prolong the half-life of the mRNA, and stemloop secondary structures were broken to improve mRNA stability and ribosomal binding. Five internal ribosome binding sites were removed to prevent any aberrant translation from within the coding sequence. Finally, six restriction sites involved in BioBrick assembly were removed. The resulting codonoptimized gene was designated mpusfus. Details of codon optimization, native and optimized sequences can be found in the Supporting Information.

Generation of Transgenic Synechococcus sp. PCC 7002 Strains. Synthetic ribosome binding sites were designed using RBS calculator ${ }^{33}$ and added directly upstream of the transgene during PCR amplification (primer sequences can be found in Table S2). Following sequence confirmation in the pGEM-T Easy vector (Promega, UK ), the amplified DNA (RBS + gene) was cloned into the $\mathrm{pAQ} 1 \mathrm{BB}$ vector, downstream of the $\mathrm{P}_{\text {cpcB594 }}$ promoter. The synthetic expression constructs were integrated into the Synechococcus genome by natural transformation. Transformation efficiency was optimized by varying either amount of DNA $(1-25 \mu \mathrm{g})$ or incubation time (1-3 days) prior to plating on selective media (Figure S1), and the following optimized transformation protocol was used: $1.5 \mathrm{~mL}$ culture $\left(\mathrm{OD}_{730} 1\right)$ was combined with $10 \mu \mathrm{g}$ circular plasmid DNA and incubated for $72 \mathrm{~h}$ under standard growth conditions with minimal sparging. Cells were plated on solid A+ medium with $1.5 \% \mathrm{w} / \mathrm{v}$ agar and $50 \mu \mathrm{g} / \mathrm{mL}$ spectinomycin. Single colonies appeared after 5-7 days. Individual colonies were isolated and grown for characterization. Genomic DNA was isolated using phenol-chloroform extraction, ${ }^{48}$ and the correct insertion of the synthetic expression constructs were verified by PCR amplification using primers pAQ1BB-seq-F (5'-CACATGAGAATTTGTCCAG-3') and pAQ1BB-seq-R ( $5^{\prime}$-CCTTTCGGGCTTTGTTAG-3') and sequencing.

Synechococcus sp. PCC 7002 Growth. Synechococcus sp. PCC 7002 cultures were grown in $\mathrm{A}+$ medium $^{18}$ (containing $300 \mathrm{mM} \mathrm{NaCl}$ ) at $30{ }^{\circ} \mathrm{C}$ with photoperiod $16 \mathrm{~h} / 8 \mathrm{~h}$ light/dark, light intensity $150 \mu \mathrm{mol}$ photons $\mathrm{m}^{-2} \mathrm{~s}^{-1}$ and sparged with humidified ambient air (standard conditions). Cultures of transformed Synechococcus were supplemented with $25 \mu \mathrm{g} / \mathrm{mL}$ spectinomycin. For mannitol analyses, cultures were grown to $\mathrm{OD}_{730} 1-3$, diluted to $\mathrm{OD}_{730} 1$ and $1.3 \mathrm{~mL}$ was inoculated to $400 \mathrm{~mL} \mathrm{~A}+$ medium. At regular intervals throughout culture 
growth, $\mathrm{OD}_{730}$ was measured and $20 \mathrm{~mL}$ of culture were harvested by centrifugation at $4000 \mathrm{~g}$ for $15 \mathrm{~min}$. Supernatants were transferred to fresh tubes and stored at $-20^{\circ} \mathrm{C}$. Cells were resuspended in $3 \mathrm{~mL}$ fresh Medium $\mathrm{A}+$, transferred to a fresh 15 $\mathrm{mL}$ Falcon tube, centrifuged at $4000 \mathrm{~g}$ for $15 \mathrm{~min}$ at $4{ }^{\circ} \mathrm{C}$, supernatants were discarded and pellets were frozen at $-20^{\circ} \mathrm{C}$. For subsequent analysis, cells were freeze-dried overnight at -50 ${ }^{\circ} \mathrm{C}$ and $0.13 \mathrm{mbar}$, and resuspended in $1 \mathrm{~mL}$ of Tris- $\mathrm{HCl} 10 \mathrm{mM}$ $\mathrm{pH}$ 8.0. After five freeze-thaw cycles, cell suspensions were sonicated $(5 \times 30 \mathrm{~s})$ using an ultrasonic processor VC50 sonicator (Jencons), and cell debris pelleted by centrifugation 15 $\mathrm{min}$ at $12500 \mathrm{~g}$. Aliquots of the cell lysates were used for determination of intracellular mannitol concentration.

Generation of Transgenic E. coli Strain. Mpusfus DNA without the initial start codon was amplified from the plasmid carrying the codon-optimized gene with the forward primer $5^{\prime}$ GGGGGGGGATCCAATAGTGCCGTGACCATCAGCC-3' (BamHI restriction site underlined) and the reverse primer $5^{\prime}$ CCCCCCGAATTCTTAGCGGGGATTGGGATCTTC-3' (EcoRI restriction site underlined). The PCR fragment obtained was cloned into the vector pFO4 as previously described, ${ }^{49}$ producing the plasmid pMPUSFUS and allowing the recombinant proteins to be fused to a six-histidine tag at their $\mathrm{N}$ terminus. This plasmid was subsequently transformed in E. coli expression strain BL21 (DE3). For gene induction and functional assays, three distinct recombinant clones were precultured in $5 \mathrm{~mL}$ of $\mathrm{M} 9$ medium supplemented with $10 \mathrm{~g} /$ $\mathrm{L}$ of glucose and $0.1 \mathrm{~g} / \mathrm{L}$ ampicillin overnight at $37^{\circ} \mathrm{C}$ and 200 $\mathrm{rpm}$. The following day, new cultures were started at $\mathrm{OD}_{600} 0.1$ under the same conditions. When $\mathrm{OD}_{600} 0.5$ was attained, cultures were divided into two tubes, and isopropyl $\beta$-D-1thiogalactopyranoside (IPTG) was added in one of them at a final concentration of $1 \mathrm{mM}$. Incubation was carried out for $20 \mathrm{~h}$ at $25^{\circ} \mathrm{C}$ and $200 \mathrm{rpm}$, and cultures reached a final $\mathrm{OD}_{600}$ of $2.5-$ 3.0 in both induced and noninduced conditions. Samples were then harvested by centrifugation at $3500 \mathrm{~g}$ for $10 \mathrm{~min}$. Supernatants and cell pellets were frozen individually at -20 ${ }^{\circ} \mathrm{C}$ for downstream analyses. To determine intracellular mannitol concentration, cells were resuspended in $600 \mu \mathrm{L}$ of buffer Tris- $\mathrm{HCl} 25 \mathrm{mM} \mathrm{pH} 7.5$ and $\mathrm{NaCl} 200 \mathrm{mM}$, and disrupted by four pulses of $15 \mathrm{~s}$ of sonication using an ultrasonic processor VC50 sonicator (Jencons). Cell debris were pelleted by centrifugation at $14000 \mathrm{~g}$ and $4{ }^{\circ} \mathrm{C}$ for $20 \mathrm{~min}$; supernatants were used for mannitol analysis and considered as soluble fractions for protein gel and Western-blot analysis. Proteins were quantified using the Pierce Coomassie Plus Assay Kit (Thermo Fisher Scientific) according to manufacturer's instructions. Three $\mu \mathrm{g}$ of protein of each fractions were loaded on a $10 \%$ Mini-PROTEAN TGX Precast Protein Gels (Biorad). After separation, proteins were transferred to nitrocellulose membrane using the iBlot 2 Dry blotting System (Thermo Fisher Scientific). Recombinant proteins were visualized with a conjugated monoclonal anti-polyhistidine-peroxidase antibody (Sigma-Aldrich) detected by the SuperSignal West Pico Chemiluminescent Substrate (Thermo Fisher Scientific).

Measurement of GFP. RBS and promoter activity was assessed in transgenic cyanobacteria using a GFP reporter encoded by BioBrick part BBa_E0040. Cultures were harvested for GFP measurements at the same point during the diurnal cycle and, when appropriate, growth stage. Optical density was adjusted to $\mathrm{OD}_{730} 0.25-0.30$ in fresh $\mathrm{A}+$ media and fluorescence was measured using a LS 55 Luminescence Spectrophotometer
(PerkinElmer, UK) using $480 \mathrm{~nm}$ excitation and $514 \mathrm{~nm}$ emission wavelengths.

Mannitol Analysis. To determine mannitol production, 50-200 $\mu \mathrm{L}$ aliquots of culture media or bacterial extracts were analyzed with the D-Mannitol/L-Arabitol assay kit (K-MANOL, Megazyme) according to the manufacturer's instructions.

\section{ASSOCIATED CONTENT}

\section{Supporting Information}

The Supporting Information is available free of charge on the ACS Publications website at DOI: 10.1021/acssynbio.8b00238.

Details for codon optimization of Micromonas pusilla $\mathrm{M} 1 \mathrm{PDH} / \mathrm{M} 1 \mathrm{Pase}$ fusion gene (PDF)

List of primers used for $\mathrm{pAQ} 1 \mathrm{BB}$ construction and to generate RBS + gene constructs (XLSX)

Figure S1 (PDF)

\section{AUTHOR INFORMATION}

\section{Corresponding Author}

*E-mail: thierry.tonon@york.ac.uk.

ORCID $\odot$

Thierry Tonon: 0000-0002-1454-6018

\section{Author Contributions}

MAM, AA, and TT conceived the study. Experimental data were generated by MAM, SS, and TT. All authors analyzed data. MAM, AA, and TT wrote the manuscript.

Notes

The authors declare no competing financial interest.

\section{ACKNOWLEDGMENTS}

We are grateful to John Golbeck and Donald Bryant (Pennsylvania State University, State College, PA) for providing the Synechococcus sp. PCC 7002 strain and the transformation vector $\mathrm{pAQ1EX.} \mathrm{This} \mathrm{work} \mathrm{was} \mathrm{funded} \mathrm{by} \mathrm{the} \mathrm{Biotechnology}$ and Biological Sciences Research Council (BB/R505195/1 and IBCarb-BIV-0316), and by the Leverhulme Trust (grant number RPG-2015-102). The funders had no role in study design, data collection and interpretation, or the decision to submit the work for publication.

\section{REFERENCES}

(1) Singh, R., Parihar, P., Singh, M., Bajguz, A., Kumar, J., Singh, S., Singh, V. P., Prasad, S. M., et al. (2017) Uncovering potential applications of cyanobacteria and algal metabolites in biology, agriculture and medicine: Current status and future prospects. Front. Microbiol. 8, 515.

(2) Gustavsson, M., and Lee, S. Y. (2016) Prospects of microbial cell factories developed through systems metabolic engineering. Microb. Biotechnol. 9, 610-617.

(3) Jeschek, M., Gerngross, D., and Panke, S. (2017) Combinatorial pathway optimization for streamlined metabolic engineering. Curr. Opin. Biotechnol. 47, 142-151.

(4) Chae, T. U., Choi, S. Y., Kim, J. W., Ko, Y. S., and Lee, S. Y. (2017) Recent advances in systems metabolic engineering tools and strategies. Curr. Opin. Biotechnol. 47, 67-82.

(5) Nielsen, J., Archer, J., Essack, M., Bajic, V. B., Gojobori, T., and Mijakovic, I. (2017) Building a bio-based industry in the Middle East through harnessing the potential of the Red Sea biodiversity. Appl. Microbiol. Biotechnol. 101, 4837-4851.

(6) Jensen, M. K., and Keasling, J. D. (2015) Recent applications of synthetic biology tools for yeast metabolic engineering. FEMS Yeast Res. $15,1-10$. 
(7) Miles, E. W., Rhee, S., and Davies, D. R. (1999) The molecular basis of substrate channeling. J. Biol. Chem. 274, 12193-12196.

(8) Dai, Y., Meng, Q., Mu, W., and Zhang, T. (2017) Recent advances in the applications and biotechnological production of mannitol. J. Funct. Foods 36, 404-409.

(9) Oddo, E., Saiano, F., Alonzo, G., and Bellini, E. (2002) An investigation of the seasonal pattern of mannitol content in deciduous and evergreen species of the oleaceae growing in northern Sicily. Ann. Bot. 90, 239-243.

(10) Saha, B. C., and Racine, F. M. (2011) Biotechnological production of mannitol and its applications. Appl. Microbiol. Biotechnol. 89, 879-891.

(11) Hays, S. G., and Ducat, D. C. (2015) Engineering cyanobacteria as photosynthetic feedstock factories. Photosynth. Res. 123, 285-295.

(12) Hagemann, M., and Hess, W. R. (2018) Systems and synthetic biology for the biotechnological application of cyanobacteria. Curr. Opin. Biotechnol. 49, 94-99.

(13) Cassier-Chauvat, C., Dive, V., and Chauvat, F. (2017) Cyanobacteria: photosynthetic factories combining biodiversity, radiation resistance, and genetics to facilitate drug discovery. Appl. Microbiol. Biotechnol. 101, 1359-1364.

(14) Al-Haj, L., Lui, Y. T., Abed, R. M., Gomaa, M. A., and Purton, S. (2016) Cyanobacteria as chassis for industrial biotechnology: Progress and prospects. Life 6, E42.

(15) Minas, K., Karunakaran, E., Bond, T., Gandy, C., Honsbein, A., Madsen, M. A., Amezaga, J., Amtmann, A., Templeton, M. R., Biggs, C. A., and Lawton, L. (2015) Biodesalination: an emerging technology for targeted removal of $\mathrm{Na}^{+}$and $\mathrm{Cl}^{-}$from seawater by cyanobacteria. Desalin. Water Treat. 55, 2647-2668.

(16) Nomura, C. T., Sakamoto, T., and Bryant, D. A. (2006) Roles for heme-copper oxidases in extreme high-light and oxidative stress response in the cyanobacterium Synechococcus sp. PCC 7002. Arch. Microbiol. 185, 471-479.

(17) Van Baalen, C. (1962) Studies on marine blue-green algae. Bot. Mar. 4, 129-139.

(18) Stevens, S. E., and Porter, R. D. (1980) Transformation in Agmenellum quadruplicatum. Proc. Natl. Acad. Sci. U. S. A. 77, 60526056.

(19) Frigaard, N. U., Sakuragi, Y., and Bryant, D. A. (2004) Gene inactivation in the cyanobacterium Synechococcus sp. PCC 7002 and the green sulfur bacterium Chlorobium tepidum using in vitro-made DNA constructs and natural transformation. Methods Mol. Biol. 274, 325340.

(20) Ruffing, A. M., Jensen, T. J., and Strickland, L. M. (2016) Genetic tools for advancement of Synechococcus sp. PCC 7002 as a cyanobacterial chassis. Microb. Cell Fact. 15, 190.

(21) Markley, A. L., Begemann, M. B., Clarke, R. E., Gordon, G. C., and Pfleger, B. F. (2015) Synthetic biology toolbox for controlling gene expression in the cyanobacterium Synechococcus sp. strain PCC 7002. ACS Synth. Biol. 4, 595-603.

(22) Zess, E. K., Begemann, M. B., and Pfleger, B. F. (2016) Construction of new synthetic biology tools for the control of gene expression in the cyanobacterium Synechococcus sp. strain PCC 7002. Biotechnol. Bioeng. 113, 424-432.

(23) Jacobsen, J. H., and Frigaard, N. U. (2014) Engineering of photosynthetic mannitol biosynthesis from $\mathrm{CO}_{2}$ in a cyanobacterium. Metab. Eng. 21, 60-70.

(24) Michel, G., Tonon, T., Scornet, D., Cock, J. M., and Kloareg, B. (2010) Central and storage carbon metabolism of the brown alga Ectocarpus siliculosus: insights into the origin and evolution of storage carbohydrates in Eukaryotes. New Phytol. 188, 67-81.

(25) Bonin, P., Groisillier, A., Raimbault, A., Guibert, A., Boyen, C., and Tonon, T. (2015) Molecular and biochemical characterization of mannitol-1-phosphate dehydrogenase from the model brown alga Ectocarpus sp. Phytochemistry 117, 509-20.

(26) Groisillier, A., Shao, Z., Michel, G., Goulitquer, S., Bonin, P., Krahulec, S., Nidetzky, B., Duan, D., Boyen, C., and Tonon, T. (2014) Mannitol metabolism in brown algae involves a new phosphatase family. J. Exp. Bot. 65, 559-570.
(27) Rousvoal, S., Groisillier, A., Dittami, S. M., Michel, G., Boyen, C., and Tonon, T. (2011) Mannitol-1-phosphate dehydrogenase activity in Ectocarpus siliculosus, a key role for mannitol synthesis in brown algae. Planta 233, 261-273.

(28) Tonon, T., Li, Y., and McQueen-Mason, S. (2017) Mannitol biosynthesis in algae: more widespread and diverse than previously thought. New Phytol. 213, 1573-1579.

(29) Shetty, R. P., Endy, D., and Knight, T. F. J. (2008) Engineering BioBrick vectors from BioBrick parts. J. Biol. Eng. 2, 5.

(30) Xu, Y., Alvey, R. M., Byrne, P. O., Graham, J. E., Shen, G., and Bryant, D. A. (2011) Expression of genes in cyanobacteria: adaptation of endogenous plasmids as platforms for high-level gene expression in Synechococcus sp. PCC 7002. Methods Mol. Biol. 684, 273-293.

(31) Zhou, J., Zhang, H., Meng, H., Zhu, Y., Bao, G., Zhang, Y., Li, Y., and Ma, Y. (2015) Discovery of a super-strong promoter enables efficient production of heterologous proteins in cyanobacteria. Sci. Rep. 4, 4500 .

(32) Reeve, B., Hargest, T., Gilbert, C., and Ellis, T. (2014) Predicting translation initiation rates for designing synthetic biology. Front. Bioeng. Biotechnol. 2, 1.

(33) Salis, H. M., Mirsky, E. A., and Voigt, C. A. (2009) Automated design of synthetic ribosome binding sites to control protein expression. Nat. Biotechnol. 27, 946-950.

(34) van Baren, M. J., Bachy, C., Reistetter, E. N., Purvine, S. O., Grimwood, J., Sudek, S., Yu, H., Poirier, C., Deerinck, T. J., Kuo, A., et al. (2016) Evidence-based green algal genomics reveals marine diversity and ancestral characteristics of land plants. BMC Genomics 17, 267.

(35) Worden, A. Z., Lee, J. H., Mock, T., Rouzé, P., Simmons, M. P., Aerts, A. L., Allen, A. E., Cuvelier, M. L., Derelle, E., Everett, M. V., et al. (2009) Green evolution and dynamic adaptations revealed by genomes of the marine picoeukaryotes. Science 324, 268-272.

(36) Blatt, A., Bauch, M. E., Pörschke, Y., and Lohr, M. (2015) A lycopene $\beta$-cyclase/lycopene $\varepsilon$-cyclase/light-harvesting complex-fusion protein from the green alga Ostreococcus lucimarinus can be modified to produce $\alpha$-carotene and $\beta$-carotene at different ratios. Plant J. 82, 582595

(37) Green, R., Hanfrey, C. C., Elliott, K. A., McCloskey, D. E., Wang, X., Kanugula, S., Pegg, A. E., and Michael, A. J. (2011) Independent evolutionary origins of functional polyamine biosynthetic enzyme fusions catalyzing de novo diamine to triamine formation. Mol. Microbiol. 81, 1109-1124.

(38) Yoshida, T., Claverie, J.-M., and Ogata, H. (2011) Mimivirus reveals Mre11/Rad50 fusion proteins with a sporadic distribution in eukaryotes, bacteria, viruses and plasmids. Virol. J. 8, 427-437.

(39) Shi, H., Chen, H., Gu, Z., Zhang, H., Chen, W., and Chen, Y. Q. (2016) Application of a delta-6 desaturase with $\alpha$-linolenic acid preference on eicosapentaenoic acid production in Mortierella alpina. Microb. Cell Fact. 15, 117.

(40) Duanmu, D., Bachy, C., Sudek, S., Wong, C. H., Jiménez, V., Rockwell, N. C., Martin, S. S., Ngan, C. Y., Reistetter, E. N., van Baren, M. J., et al. (2014) Marine algae and land plants share conserved phytochrome signaling systems. Proc. Natl. Acad. Sci. U. S. A. 111, 15827-15832.

(41) Petrie, J. R., Shrestha, P., Mansour, M. P., Nichols, P. D., Liu, Q., and Singh, S. P. (2010) Metabolic engineering of omega-3 long-chain polyunsaturated fatty acids into plants using an acyl-CoA $\Delta 6$ desaturase with $\omega 3$-preference from the marine microalga Micromonas pusilla. Metab. Eng. 12, 233-240.

(42) Lada, A. G., Krick, C. F., Kozmin, S. G., Mayorov, V. I., Karpova, T. S., Rogozin, I. B., and Pavlov, Y. I. (2011) Mutator effects and mutation signatures of editing deaminases produced in bacteria and yeast. Biochemistry (Moscow) 76, 131-146.

(43) Kaup, B., Bringer-Meyer, S., and Sahm, H. (2005) D-mannitol formation from D-glucose in a whole-cell biotransformation with recombinant Escherichia coli. Appl. Microbiol. Biotechnol. 69, 397-403.

(44) Reshamwala, S. M., Pagar, S. K., Velhal, V. S., Maranholakar, V. M., Talangkar, V. G., and Lali, A. M. (2014) Construction of an efficient 
Escherichia coli whole-cell biocatalyst for D-mannitol production. J. Biosci. Bioeng. 118, 628-631.

(45) Hagemann, M. (2011) Molecular biology of cyanobacterial salt acclimation. FEMS Microbiol. Rev. 35, 87-123.

(46) Xu, Y., Guerra, L. T., Li, Z., Ludwig, M., Dismukes, G. C., and Bryant, D. A. (2013) Altered carbohydrate metabolism in glycogen synthase mutants of Synechococcus sp. strain PCC 7002: Cell factories for soluble sugars. Metab. Eng. 16, 56-67.

(47) Gudmundsson, S., and Nogales, J. (2015) Cyanobacteria as photosynthetic biocatalysts: a systems biology perspective. Mol. BioSyst. $11,60-70$.

(48) Tamagnini, P., Troshina, O., Oxelfelt, F., Salema, R., and Lindblad, P. (1997) Hydrogenases in Nostoc sp. strain PCC 73102, a strain lacking a bidirectional enzyme. Appl. Environ. Microbiol. 63, 1801-1807.

(49) Groisillier, A., Hervé, C., Jeudy, A., Rebuffet, E., Pluchon, P. F., Chevolot, Y., Flament, D., Geslin, C., Morgado, I. M., Power, D., et al. (2010) MARINE-EXPRESS: taking advantage of high throughput cloning and expression strategies for the post-genomic analysis of marine organisms. Microb. Cell Fact. 9, 45. 